\title{
Recurrence Relations for Single and Product Moments of Generalized Order Statistics from Extreme Value Distribution
}

\author{
Kamal Nain Kapoor* \\ Hindu College, University of Delhi, Delhi, India \\ *Corresponding author: kamal.180968@gmail.com
}

Received January 08, 2014; Revised March 04, 2014; Accepted March 13, 2014

\begin{abstract}
In this paper, we establish some recurrence relations satisfied by single and product moments of Generalized Order Statistics from Extreme Value Distribution. These recurrence relations are independent of left truncated point and therefore are also applicable for Logistic as well as for half Logistic distributions studied in Balakrishnan (1985) and Saran and Pandey (2012). For a particular case these results verify the corresponding results of Saran and Pandey (2004) and Kumar (2010).
\end{abstract}

Keywords: order statistics, record values, generalized order statistics, single moment, product moments, recurrence relations, extreme value distribution

Cite This Article: Kamal Nain Kapoor, "Recurrence Relations for Single and Product Moments of Generalized Order Statistics from Extreme Value Distribution.” American Journal of Applied Mathematics and Statistics, vol. 2, no. 1 (2014): 77-82. doi: 10.12691/ajams-2-2-5.

\section{Introduction}

Generalized order statistics (GOS) have been introduced and extensively studied in Kamps (1995 a,b) as a unified theoretical set-up which contains a variety of models of ordered random variables with different interpretations. Examples of such models are: Ordinary order statistics, Sequential order statistics, Progressive type II censored order statistics, Record values, $\mathrm{k}^{\text {th }}$ record value and Pfeifer's records. There is no natural interpretation of generalized order statistics in terms of observed random samples but these models can be effectively applied in life testing and reliability analysis, medical and life time data, and models related to software reliability analysis, etc. The common approach makes it possible to define several distributional properties at once. The structural similarities of these models are based on the similarity of their joint density function.

\section{Standard Extreme Value Distribution}

A random variable $\mathrm{X}$ is said to have an standard extreme value distribution if its probability density function is of the form

$$
f(x)=\alpha e^{\alpha x} \times e^{-e^{\alpha x}}, \quad-\infty<x<\infty, \alpha>0
$$

and the cumulative distribution is given by

$$
F(x)=1-e^{-e^{\alpha x}}, \quad-\infty<x<\infty, \alpha>0
$$

The extreme value distribution is used in the analysis of data concerning floods, extreme sea level and air pollution problems.

The cumulative distribution function and probability density function of random variable $\mathrm{X}$, respectively, takes the form

$$
\begin{gathered}
f(x)=\left(\sum_{j=0}^{\infty} \frac{\alpha^{j+1} x^{j}}{j !}\right)(1-F(x)) . \\
\frac{f(x)}{1-F(x)}=\left(\sum_{j=0}^{\infty} \frac{\alpha^{j+1} x^{j}}{j !}\right)=\sum_{j=0}^{\infty} \alpha_{j} x^{j}, \text { where } \alpha_{j}=\frac{\alpha^{j+1}}{j !}
\end{gathered}
$$

The mathematical form of pdf, as given in (2.3), is very useful to derive the expression for recurrence relations for single and product moments of GOS.

\section{Generalized Order Statistics}

Let $\left\{X_{n}, n \geq 1\right\}$ be a sequence of absolutely continuous, independent and identically distributed random variables with cdf $F(x)=P(X \leq x)$ and pdf $\mathrm{f}(\mathrm{x})$. Assume $\mathrm{k}>0$, $n \in\{2,3, \ldots\}, \quad \tilde{m}=\left(m_{1}, m_{2}, \ldots, m_{n-1}\right) \in R^{n-1} \quad$, $M_{r}=\sum_{j=r}^{n-1} m_{j}$, such that $\gamma_{r}=k+n-r+M_{r}>0$ for all $r \in\{1,2, \ldots, n-1\}$. Then $X(r, n, \tilde{m}, k), \mathrm{r}=1,2, \ldots, \mathrm{n}$, are called GOS if their joint pdf is given by 


$$
\begin{aligned}
& f^{X(1, n, \tilde{m}, k), X(2, n, \tilde{m}, k), \ldots, X(n, n, \tilde{m}, k)}\left(x_{1}, x_{2}, \ldots, x_{n}\right) \\
& =k\left(\prod_{j=1}^{n-1} \gamma_{j}\right)\left(\prod_{i=1}^{n-1}\left(1-F\left(x_{i}\right)\right)^{m_{i}} f\left(x_{i}\right)\right) \\
& \quad \times\left(1-F\left(x_{n}\right)\right)^{k-1} f\left(x_{n}\right),
\end{aligned}
$$

where $F^{-1}(0+)<x_{1} \leq x_{2} \leq \ldots \leq x_{n}<F^{-1}(1)$.

By choosing appropriate values of parameters, we get the distribution of a few very common statistics as shown in the table given below.

\begin{tabular}{|c|c|c|}
\hline S.No. & Choice of parameters for $\mathrm{i}=1,2, \ldots, \mathrm{n}$ & GOS becomes \\
\hline 1 & $\gamma_{i}=n-i+1, m_{1}=m_{2}=\ldots=m_{n-1}=0$ and $\mathrm{k}=1$ & Joint distribution of $\mathrm{n}$ order statistics \\
\hline 2 & $\gamma_{i}=k, m_{1}=m_{2}=\ldots=m_{n-1}=-1, k \in N$ & $\mathrm{k}^{\text {th }}$ record value \\
\hline 3 & $\gamma_{i}=(n-i+1) \alpha_{i}, \alpha_{i}>0$ & Sequential order statistics \\
\hline 4 & $\gamma_{i}=\alpha-i+1, \alpha>0$ & Order statistics with non integer sample size \\
\hline 5 & $\gamma_{i}=\beta_{i}, \beta_{i}>0$ & Pfeifer's record values \\
\hline 6 & $m_{i} \in N_{o}, k \in N$ & Progressively type-II right censored order statistics \\
\hline
\end{tabular}

The joint pdf of first $r$, GOS is given by:

$$
\begin{aligned}
& f^{X(1, n, \tilde{m}, k), X(2, n, \tilde{m}, k), \ldots, X(r, n, \tilde{m}, k)}\left(x_{1}, x_{2}, \ldots, x_{r}\right) \\
& =c_{r-1}\left(\prod_{i=1}^{r-1}\left(1-F\left(x_{i}\right)\right)^{m_{i}} f\left(x_{i}\right)\right) \\
& \quad \times\left(1-F\left(x_{r}\right)\right)^{k+n-r+M_{r}-1} f\left(x_{r}\right),
\end{aligned}
$$

where, $F^{-1}(0+)<x_{1} \leq x_{2} \leq \ldots \leq x_{r}<F^{-1}(1)$.

We now consider two cases:

Case I: $\mathrm{m}_{1}=\mathrm{m}_{2}=\ldots=\mathrm{m}_{\mathrm{n}-1}=\mathrm{m}$

Case II: $\gamma_{i} \neq \gamma_{j} ; \mathrm{i} \neq \mathrm{j}, \mathrm{i}, \mathrm{j}=1,2, \ldots, \mathrm{n}-1$.

For case I, the GOS will be denoted by $\mathrm{X}(\mathrm{r}, \mathrm{n}, \mathrm{m}, \mathrm{k})$. The pdf of $\mathrm{X}(\mathrm{r}, \mathrm{n}, \mathrm{m}, \mathrm{k})$ is given by

$$
\begin{aligned}
& f^{X(r, n, m, k)}(x) \\
= & \frac{c_{r-1}}{(r-1) !}(1-F(x))^{\gamma_{r}-1} f(x) g_{m}^{r-1}(F(x)), \quad x \in R
\end{aligned}
$$

and the joint pdf of $\mathrm{X}(\mathrm{r}, \mathrm{n}, \mathrm{m}, \mathrm{k})$ and $\mathrm{X}(\mathrm{s}, \mathrm{n}, \mathrm{m}, \mathrm{k}), 1 \leq \mathrm{r}<$ $\mathrm{s} \leq \mathrm{n}$ is given by:

$$
\begin{aligned}
& f^{X(r, n, m, k), X(s, n, m, k)}(x, y) \\
= & \frac{c_{s-1}}{(r-1) !(s-r-1) !}\left((1-F(x))^{m} f(x)\right) \\
& \cdot g_{m}^{r-1}(F(x))\left[h_{m}(F(y))-h_{m}(F(x))\right]^{s-r-1} \\
& \cdot(1-F(y))^{\gamma s^{-1}} f(y), x<y,
\end{aligned}
$$

where, $\quad c_{r-1}=\prod_{j=1}^{r} \gamma_{j}, \gamma_{j}=k+(n-j)(m+1), r=1,2, \ldots, n$, $g_{m}(x)=h_{m}(x)-h_{m}(0), x \in(0,1)$

and

$$
h_{m}(x)= \begin{cases}-\frac{(1-x)^{m+1}}{m+1}, & m \neq-1 \\ -\log (1-x), & m=-1 .\end{cases}
$$

For case II, the pdf of $X(r, n, \tilde{m}, k)$ is given by

$$
\begin{aligned}
& f^{X(r, n, \tilde{m}, k)}(x) \\
= & c_{r-1} f(x) \sum_{i=1}^{r} a_{i}(r)(1-F(x))^{\gamma_{i}-1} f(x), x \in R .
\end{aligned}
$$

Also, the joint pdf of $X(r, n, \tilde{m}, k)$ and $X(s, n, \tilde{m}, k), 1 \leq \mathrm{r}<\mathrm{s} \leq \mathrm{n}$ is given by

$$
\begin{aligned}
& f^{X(r, n, \tilde{m}, k), X(s, n, \tilde{m}, k)}(x, y) \\
= & c_{s-1}\left\{\sum_{i=r+1}^{s} a_{i}^{r}(s)\left(\frac{1-F(y)}{1-F(x)}\right)^{\gamma_{i}}\right\} \\
& \times\left\{\sum_{i=1}^{r} a_{i}(r)(1-F(x))^{\gamma_{i}}\right\} \frac{f(x)}{1-F(x)} \frac{f(y)}{1-F(y)},
\end{aligned}
$$

Where, $c_{s-1}=\prod_{j=1}^{s} \gamma_{j}, \gamma_{j}=k+n-j+M_{j}, s=1,2, \ldots, n$.

Further it can be proved that

(i) $a_{i}(r)=\prod_{j(\neq i)=1}^{r}\left(\gamma_{j}-\gamma_{i}\right)^{-1}, 1 \leq i \leq r \leq n$

(ii) $a_{i}^{r}(s)=\prod_{j(\neq i)=r+1}^{s}\left(\gamma_{j}-\gamma_{i}\right)^{-1}, r+1 \leq i \leq s \leq n$.

(iii) $a_{i}(r)=\left(\gamma_{r+1}-\gamma_{i}\right) a_{i}(r+1)$

(iv) $c_{r}=c_{r-1} \gamma_{r+1}$

(v) $\sum_{i=1}^{r+1} a_{i}(r+1)=0$

(vi)

$$
\sum_{i=1}^{r} a_{i}(r)(1-F(x))^{\gamma_{i}}=\frac{(1-F(x))^{\gamma_{r}}}{(r-1) !} g_{m}^{r-1}(F(x))
$$

(vii)

$$
\begin{aligned}
& \sum_{i=r+1}^{s} a_{i}^{r}(s)\left(\frac{1-F(y)}{1-F(x)}\right)^{\gamma_{i}} \\
= & \frac{(1-F(x))^{-(m+1)(s-r-1)}}{(s-r-1) !}\left(\frac{1-F(y)}{1-F(x)}\right)^{\gamma_{s}} \\
& \times\left(h_{m}(F(y))-h_{m}(F(x))\right)^{s-r-1}
\end{aligned}
$$

The moments of order statistics have generated considerable interest in the recent years. The expressions for several recurrence relations and identities satisfied by single as well as product moments of order statistics have been obtained by several authors in the past. These 
relations help in reducing the quantum of computations involved. Joshi (1978, 1982) established recurrence relations for exponential distribution with unit mean and were further extended by Balakrishnan and Joshi (1984) for doubly truncated exponential distribution. For linearexponential distribution, Balakrishnan and Malik(1986) derived the similar type of relations which were extended to doubly truncated linear exponential distribution by Mohie El-Din et al. (1997) and Saran and Pushkarna (1999). Nain (2010 a, b) obtained recurrence relations for ordinary order statistics and $\mathrm{k}^{\text {th }}$ record values from $\mathrm{p}^{\text {th }}$ order exponential and generalized weibull distributions, respectively.

The recurrence relations for the moments of generalized order statistics based on non identically distributed random variables were developed by Kamps (1995 a, b). Pawlas and Szynal (2001) obtained recurrence relations for single and product moments of generalized order statistics from Pareto, generalized Pareto and Burr distributions. Saran and Pandey (2004, 2009) obtained recurrence relations for single and product moments of generalized order statistics from linear-exponential and Burr distributions. Saran and Nain (2012) obtained recurrence relations for single and product moments generalized order statistics from doubly truncated $\mathrm{p}^{\text {th }}$ order exponential distribution. Saran and Nain (2013) also obtained explicit expressions for single and product moments of Generalized Order Statistics from a new class of exponential distribution.

In this paper, we have established recurrence relations for single and product moments of GOS from Extreme Value Distribution. This distribution has many applications in analysis of data concerning floods, extreme sea levels and air pollution problems.

The results so obtained are generalized versions of some of the recurrence relations obtained by Kumar (2010), Saran and Pandey (2004).

\section{Notations}

For $\mathrm{n}=1, \quad 2, \quad 3, \quad \ldots, \quad 1 \leq r<s \leq n, k \geq 1 \quad$ and $u, v \in\{0,1,2, \ldots\}$, we denote by

(i) $\mu_{r: m, n, k}^{u}=E\left(\mathrm{X}^{u}(\mathrm{r}, \mathrm{n}, \mathrm{m}, \mathrm{k})\right)$

(ii) $\mu_{r, s: m, n, k}^{u, v}=E\left(\mathrm{X}^{u}(\mathrm{r}, \mathrm{n}, \mathrm{m}, \mathrm{k}) \mathrm{X}^{v}(\mathrm{~s}, \mathrm{n}, \mathrm{m}, \mathrm{k})\right)$

(iii) $\mu_{r: \tilde{m}, n, k}^{u}=E\left(\mathrm{X}^{u}(\mathrm{r}, \mathrm{n}, \tilde{\mathrm{m}}, \mathrm{k})\right)$

(iv) $\mu_{r, s: \tilde{m}, n, k}^{u, v}=E\left(\mathrm{X}^{u}(\mathrm{r}, \mathrm{n}, \tilde{\mathrm{m}}, \mathrm{k}) \mathrm{X}^{v}(\mathrm{~s}, \mathrm{n}, \tilde{\mathrm{m}}, \mathrm{k})\right)$

\section{Recurrence Relations For Single and Product Moments}

Case I: $m_{1}=m_{2}=\ldots=m_{n-1}=m$

\section{Theorem 1.}

For $\mathrm{n}=1,2, \quad 3, \quad \ldots, \quad 1 \leq r<s \leq n, k \geq 1$ and $u, v \in\{0,1,2, \ldots\}$

(a)

$\mu_{r: m, n, k}^{u}=\alpha \gamma_{r} \sum_{j=0}^{\infty} \frac{\alpha^{j}}{u+j+1}\left[\begin{array}{c}u+j+1 \\ u r: m, n, k\end{array}-\mu_{r-1: m, n, k}^{u+j+1}\right]$ (4.1) (b)

$$
\begin{aligned}
& \mu_{r, s: m, n, k}^{u, v}(r, s) \\
= & \alpha \gamma_{s} \sum_{j=0}^{\infty} \frac{\alpha^{j}}{v+j+1}\left[\mu_{r, s: m, n, k}^{u, v+j+1}-\mu_{r, s-1: m, n, k}^{u, v+j+1}\right]
\end{aligned}
$$

Proof (a):

The $\mathrm{u}^{\text {th }}$ order moment of $\mathrm{X}(\mathrm{r}, \mathrm{n}, \mathrm{m}, \mathrm{k})$ is given by

$$
\begin{aligned}
& \mu_{r: m, n, k}^{u} \\
= & \frac{c_{r-1}}{(r-1) !} \int_{\beta}^{\infty} x^{u}(1-F(x))^{\gamma_{r}-1} f(x) g_{m}^{r-1}(F(x)) d x .
\end{aligned}
$$

Substituting $f(x)$ from (2.3) we have

$\mu_{r: m, n, k}^{u}$

$=\frac{c_{r-1}}{(r-1) !} \sum_{j=0}^{\infty} \alpha_{j} \int_{\beta}^{\infty} x^{u+j}(1-F(x))^{\gamma_{r}} g_{m}^{r-1}(F(x)) d x$.

Integrating by parts, taking $\mathrm{x}^{\mathrm{u}+\mathrm{j}}$ as the part to be integrated, we obtain

$$
\begin{aligned}
\mu_{r: m, n, k}^{u} & =\frac{c_{r-1}}{(r-1) !} \sum_{j=0}^{\infty} \frac{\alpha_{j}}{u+j+1} \\
\int_{\beta}^{\infty} x^{u+j} & +1\left\{\gamma_{r}(1-F(x))^{\gamma_{r}-1} g_{m}^{r-1}(F(x)) f(x)\right. \\
& \left.-(r-1)(1-F(x))^{\gamma_{r}+m} g_{m}^{r-2}(F(x)) f(x)\right\}
\end{aligned}
$$

On substituting $\gamma_{r}+m=\gamma_{r-1}-1, c_{r-1}=\gamma_{r} c_{r-2}$ and $\alpha_{j}=\alpha^{j+1}$ in (4.5), we shall derive the recurrence relation as stated in (4.1).

Proof (b). By definition

$$
\begin{aligned}
\mu_{r, s: m, n, k}^{u, v} & =\frac{1}{(r-1) !(s-r-1) !} \\
& \times \int_{\beta \times g_{m}^{r-1}(F(x)) J(x: v, r, s, m)}^{\infty} x^{u}\left((1-F(x))^{m} f(x)\right)
\end{aligned}
$$

where,

$$
\begin{aligned}
& J(x: v, r, s, m) \\
= & c_{s}-1 \int_{x}^{\infty} y^{v}\left[h_{m}(F(y))-h_{m}(F(x))\right]^{s-r-1} d y
\end{aligned}
$$

Substituting $\mathrm{f}(\mathrm{y})$ from (2.3) we have:

$$
\begin{aligned}
J(x: v, r, s, m) & c_{s-1} \sum_{j=0}^{\infty} \alpha_{j} \int_{x}^{\infty} y^{v+j}\left[\begin{array}{c}
h_{m}(F(y)) \\
-h_{m}(F(x))
\end{array}\right]^{s-r-1} d y . \\
&
\end{aligned}
$$

Integrating by parts, taking $\mathrm{y}^{\mathrm{v}+\mathrm{j}}$ as the part to be integrated, we obtain: 


$$
\begin{aligned}
J(x: v, r, s, m) & =c_{s-1} \sum_{j=0}^{\infty} \frac{\alpha_{j}}{v+j+1} \\
\int_{x}^{\infty} y^{v+j+1} & \left\{\gamma_{s}\left[h_{m}(F(y))-h_{m}(F(x))\right]^{s-r-1}\right. \\
\times & (1-F(y))^{\gamma_{s}-1} f(y)-(s-r-1) \\
\times & {\left[h_{m}(F(y))-h_{m}(F(x))\right]^{s-r-2} } \\
\times & \left.(1-F(y))^{\gamma_{s}+m} f(y)\right\} d y
\end{aligned}
$$

After using $\gamma_{s}+m=\gamma_{s-1}-1$ and $c_{s-1}=\gamma_{s} c_{s-2}$, we get:

$$
\begin{aligned}
& J(x: v, r, s, m) \\
= & \gamma_{s} \sum_{j=0}^{\infty} \frac{\alpha_{j}}{v+j+1}\left\{\begin{array}{l}
J(x: v+j+1, r, s, m) \\
-(s-r-1) J(x: v+j+1, r, s-1, m)
\end{array}\right\} .
\end{aligned}
$$

On substituting $\mathrm{J}(\mathrm{x}: \mathrm{v}, \mathrm{r}, \mathrm{s}, \mathrm{m})$ so obtained in (4.6), we shall derive the recurrence relation as stated in (4.2).

Case II: $\gamma_{i} \neq \gamma_{j} ; \mathrm{i} \neq \mathrm{j}, \mathrm{i}, \mathrm{j}=1,2, \ldots, \mathrm{n}-1$.

\section{Lemma 1.}

(a)

$$
\begin{aligned}
& c_{r-1} \sum_{i=1}^{r} a_{i}(r) \gamma_{i}(1-F(x))^{\gamma_{i}} \\
&=\gamma_{r}\left[\begin{array}{c}
c_{r-1} \sum_{i=1}^{r} a_{i}(r)(1-F(x))^{\gamma_{i}} \\
-c_{r-2} \sum_{i=1}^{r-1} a_{i}(r-1)(1-F(x))^{\gamma_{i}}
\end{array}\right]
\end{aligned}
$$

(b)

$$
\begin{aligned}
& c_{s-1} \sum_{i=r+1}^{s} a_{i}^{r}(s) \gamma_{i}\left(\frac{1-F(y)}{1-F(x)}\right)^{\gamma_{i}} \\
& =\gamma_{s}\left[\begin{array}{c}
c_{s-1} \sum_{i=r+1}^{s} a_{i}^{r}(s)\left(\frac{1-F(y)}{1-F(x)}\right)^{\gamma_{i}} \\
-c_{s-2} \sum_{i=r+1}^{s-1} a_{i}^{r}(s-1)\left(\frac{1-F(y)}{1-F(x)}\right)^{\gamma_{i}}
\end{array}\right]
\end{aligned}
$$

Proof (a): Differentiating both sides of (3.9), with respect to $\mathrm{x}$, we get:

$$
\begin{aligned}
& \sum_{i=1}^{r} a_{i}(r) \gamma_{i}(1-F(x))^{\gamma_{i}-1} \\
& =\frac{1}{(r-1) !}\left[\begin{array}{l}
\gamma_{r}(1-F(x))^{\gamma_{r}-1} g_{m}^{r-1}(F(x)) \\
-(r-1)(1-F(x))^{\gamma_{r}} g_{m}^{r-2}(F(x)) g_{m}^{\prime}(F(x))
\end{array}\right] \\
& =\frac{1}{(r-1) !}\left[\begin{array}{l}
\gamma_{r}(1-F(x))^{\gamma_{r}-1} g_{m}^{r-1}(F(x)) \\
-(r-1)(1-F(x))^{\gamma_{r}+m} g_{m}^{r-2}(F(x))
\end{array}\right] \\
& \left(\because g_{m}^{\prime}(F(x))=(1-F(x))^{m}\right)
\end{aligned}
$$

$$
\begin{aligned}
& =\frac{1}{(r-1) !}\left[\begin{array}{c}
\gamma_{r}(1-F(x))^{\gamma_{r}-1} g_{m}^{r-1}(F(x)) \\
-(r-1)(1-F(x))^{\gamma_{r}-1^{-1}} g_{m}^{r-2}(F(x))
\end{array}\right] \\
& =\frac{1}{1-F(x)}\left[\begin{array}{c}
\gamma_{r} \sum_{i=1}^{r} a_{i}(r)(1-F(x))^{\gamma_{i}} \\
-\sum_{i=1}^{r-1} a_{i}(r-1)(1-F(x))^{\gamma_{i}}
\end{array}\right],
\end{aligned}
$$

which on using the relation $c_{r-1}=\gamma_{r} c_{r-2}$ leads to (4.9).

Proof (b): Differentiating both sides of (3.10), with respect to $\mathrm{y}$, we get:

$$
\begin{aligned}
& \sum_{i=r+1}^{s} a_{i}^{r}(s) \gamma_{i} \frac{(1-F(y))^{\gamma_{i}-1}}{(1-F(x))^{\gamma_{i}}} \\
& =\frac{(1-F(x))^{-(m+1)(s-r-1)}}{(s-r-1) !(1-F(x))^{\gamma_{s}}} \\
& \times\left\{\begin{array}{l}
\gamma_{s}(1-F(y))^{\gamma_{s}-1}\left(h_{m}(F(y))-h_{m}(F(x))\right)^{s-r-1} \\
-(s-r-1)(1-F(y))^{\gamma_{s}} \\
\times\left(h_{m}(F(y))-h_{m}(F(x))\right)^{s-r-2} h_{m}^{\prime}(F(y))
\end{array}\right\} \\
& =\frac{(1-F(x))^{-(m+1)(s-r-1)}}{(s-r-1) !(1-F(x))^{\gamma_{s}}} \\
& \times\left\{\begin{array}{l}
\gamma_{s}(1-F(y))^{\gamma_{s}-1}\left(h_{m}(F(y))-h_{m}(F(x))\right)^{s-r-1} \\
-(s-r-1)(1-F(y))^{\gamma_{s}+m}\left(\begin{array}{c}
h_{m}(F(y)) \\
-h_{m}(F(x))
\end{array}\right)^{s-r-2}
\end{array}\right\} \\
& \left(\because \quad h_{m}^{\prime}(F(y))=(1-F(y))^{m}\right)
\end{aligned}
$$$$
=\left\{\begin{array}{l}
\frac{\gamma_{s}(1-F(x))^{-(m+1)(s-r-1)}}{(s-r-1) !(1-F(y))} \\
\times\left(\frac{1-F(y)}{1-F(x)}\right)^{\gamma_{s}}\left(h_{m}(F(y))-h_{m}(F(x))\right)^{s-r-1} \\
-\frac{(1-F(x))^{-(m+1)(s-r-2)}}{(s-r-2) !(1-F(y))} \\
\times\left(\frac{1-F(y)}{1-F(x)}\right)^{\gamma_{s}-1}\left(h_{m}(F(y))-h_{m}(F(x))\right)^{s-r-2}
\end{array}\right\}
$$$$
=\frac{1}{(1-F(y))}\left[\begin{array}{c}
\gamma_{s} \sum_{i=r+1}^{s} a_{i}^{r}(s)\left(\frac{1-F(y)}{1-F(x)}\right)^{\gamma_{i}} \\
-\sum_{i=r+1}^{s-1} a_{i}^{r}(s-1)\left(\frac{1-F(y)}{1-F(x)}\right)^{\gamma_{i}}
\end{array}\right],
$$

which on using the relation $c_{S-1}=\gamma_{S} c_{S-2}$ leads to (4.10).

Theorem 2.

For $\mathrm{n}=1, \quad 2, \quad 3, \ldots, \quad 1 \leq r<s \leq n, k \geq 1 \quad$ and $u, v \in\{0,1,2, \ldots\}$ 
$\mu_{r: \tilde{m}, n, k}^{u}=\alpha \gamma_{r} \sum_{j=0}^{\infty} \frac{\alpha^{j}}{u+j+1}\left[\mu_{r: \tilde{m}, n, k}^{u+j+1}-\mu_{r-1: \tilde{m}, n, k}^{u+j+1}\right]$

(b)

$$
\mu_{r, s: \tilde{m}, n, k}^{u, v}=\alpha \gamma_{s} \sum_{j=0}^{\infty} \frac{\alpha^{j}}{v+j+1}\left[\begin{array}{c}
\mu_{r, s: \tilde{m}, n, k}^{u, v+j+1} \\
-\mu_{r, s-1: \tilde{m}, n, k}^{u, v+j+1}
\end{array}\right]
$$

\section{Proof (a):}

The $\mathrm{u}^{\text {th }}$ order moment of $X(r, n, \tilde{m}, k)$ is given by:

$$
\mu_{r: \tilde{m}, n, k}^{u}=c_{r-1} \int_{\beta}^{\infty} x^{u} \sum_{i=1}^{r} a_{i}(r)\left(1-F(x)^{\gamma_{i}-1} f(x) d x\right.
$$

Substituting the value of $f(x)$ from (2.3), we have

$$
\mu_{r: \tilde{m}, n, k}^{u}=c_{r-1} \sum_{j=0}^{\infty} \alpha_{j}\left\{\int_{\beta}^{\infty} x^{u+j} \sum_{i=1}^{r} a_{i}(r)\left(1-F(x)^{\gamma_{i}} d x\right\} .\right.
$$

Integrating by parts, taking $\mathrm{x}^{\mathrm{u}+\mathrm{j}}$ as the part to be integrated, we obtain:

$$
\begin{aligned}
& \mu_{r: \tilde{m}, n, k}^{u} \\
= & c_{r-1} \sum_{j=0}^{\infty} \frac{\alpha_{j}}{u+j+1} \\
\times & \left\{\int_{\beta}^{\infty} x^{u+j+1} \sum_{i=1}^{r} a_{i}(r) \gamma_{i}\left(1-F(x)^{\gamma_{i}-1} f(x)\right\}\right.
\end{aligned}
$$

After using (4.9), we shall derive the recurrence relation given in (4.11).

Proof (b): We know that

$$
\begin{aligned}
\mu_{r, s: \tilde{m}, n, k}^{u, v}=\int_{\beta}^{\infty} & x^{u} \sum_{i=1}^{r} a_{i}(r)(1-F(x))^{\gamma_{i}} \\
& \times \frac{f(x)}{1-F(x)} J(x: v, r, s, \tilde{m}) d x,
\end{aligned}
$$

$$
J(x: v, r, s, \tilde{m})
$$

where $=c_{s-1} \int_{x}^{\infty} y^{v} \sum_{i=r+1}^{s} a_{i}^{r}(s)\left(\frac{1-F(y)}{1-F(x)}\right)^{\gamma_{i}} \frac{f(y)}{1-F(y)} d y$.

Substituting $\mathrm{f}(\mathrm{y})$ from (2.3) we have:

$$
\begin{aligned}
& J(x: v, r, s, \tilde{m}) \\
= & \sum_{j=0}^{\infty} \alpha_{j}\left\{c_{s-1} \int_{x}^{\infty} y^{v+j} \sum_{i=r+1}^{s} a_{i}^{r}(s)\left(\frac{1-F(y)}{1-F(x)}\right)^{\gamma_{i}} d y\right\} .
\end{aligned}
$$

Integrating by parts, taking $\mathrm{y}^{\mathrm{v}+\mathrm{j}}$ as the part to be integrated, we obtain:

$$
\begin{aligned}
& J(x: v, r, s, \tilde{m}) \\
& =\sum_{j=0}^{\infty} \frac{\alpha_{j}}{v+j+1}\left\{c_{s-1} \int_{x}^{\infty} \times \sum_{i=r+1}^{s} a_{i}^{r}(s) \frac{\gamma_{i}(1-F(y))^{\gamma_{i}-1}}{(1-F(x))^{\gamma_{i}}} f(y) d y\right\}
\end{aligned}
$$

$$
\begin{aligned}
& \left.=\sum_{j=0}^{\infty} \frac{\alpha_{j}}{v+j+1}\left\{\int_{x \times\left[c_{s-1} \sum_{i=r+1}^{s} a_{i}^{r}(s) \frac{\gamma_{i}(1-F(y))^{\gamma_{i}}}{(1-F(x))^{\gamma_{i}}}\right]}\right]^{d y}\right\} \\
& =\gamma_{s} \sum_{j=0}^{\infty} \frac{\alpha_{j}}{v+j+1}\left\{\left[c_{s-1} \int_{x}^{\infty} y^{v+j+1} \sum_{i=r+1}^{s} a_{i}^{r}(s)\left(\frac{1-F(y)}{1-F(x)}\right)^{\gamma_{i}}\right.\right. \\
& \left.\left.-c_{s-2} \int_{x}^{\infty} y^{v+j+1} \sum_{i=r+1}^{s-1} a_{i}^{r}(s-1)\left(\frac{1-F(y)}{1-F(x)}\right)^{\gamma_{i}}\right] \frac{f(y)}{1-F(y)} d y\right\}
\end{aligned}
$$

$=\gamma_{s} \sum_{j=0}^{\infty} \frac{\alpha_{j}}{v+j+1}\left[\begin{array}{l}J(x: v+j+1, r, s, \tilde{m}) \\ -J(x: v+j+1, r, s-1, \tilde{m})\end{array}\right]$.

On substituting the above expression of $J(x: v, r, s, \tilde{m})$ in (4.16) we get

$$
\begin{aligned}
& \mu_{r, s: \tilde{m}, n, k}^{u, v} \\
& =\gamma_{s} \sum_{j=0}^{\infty} \frac{\alpha_{j}}{v+j+1} \int_{\beta}^{\infty} \quad \times\left\{\begin{array}{l}
x^{u} \\
\sum_{i=1}^{r} a_{i}(x)(1-F(x))^{\gamma_{i}} \frac{f(x)}{1-F(x)} \\
-J(x: v+j+1, r, s, \tilde{m})
\end{array}\right\} d x
\end{aligned}
$$

which on using (4.16), leads to (4.12).

\section{Conclusion}

In the study presented above, we demonstrate the recurrence relations for single and product moments of GOS from Extreme Value Distribution. These results generalize the corresponding results of Kumar (2010) and Saran and Pandey (2004).

\section{Acknowledgement}

The authors wish to thanks the referee for valuable comments, which led to an improvement in the presentation of this paper.

\section{References}

[1] Athar, H., Kheaja S. K. and Nayabuddin (2012). Expectation identities of Pareto distribution based on generalized order statistics. American Journal of Applied Mathematics and Mathematical Sciences, 1, 23-29.

[2] Balakrishnan, N. (1985). Order statistics from the Half logistic distribution. J. Statist. Comp. Simul., 20, 287-309.

[3] Balakrishnan, N. and Joshi, P.C. (1984). Product moments of order statistics from doubly truncated exponential distribution. Naval Res. Logist. Quart, 31, 27-31.

[4] Balakrishnan, N. and Malik, H. J. (1986). Order statistics from linear exponential distribution, Part I: Increasing hazard rate case. Commun. Statist. - Theor. Meth. 15, 179-203.

[5] Joshi, P. C. (1978). Recurrence relations between moments of order statistics from exponential and truncated exponential distributions. Sankhya, Ser. B, 39, 362-371. 
[6] Joshi, P. C. (1982). A note on mixed moments of order statistics from exponential and truncated exponential distributions. J. Statist. Plann. Inf., 6, 13-16.

[7] Kamps, U. (1995a). A concept of generalized order statistics. B. G. Teubner, Stuttgart.

[8] Kamps, U. (1995b). A concept of generalized order statistics. J. Statist. Plann. Inf., 48, 1-23.

[9] Kumar, D. (2010). Recurrence relations for single and product moments of generalized order statistics from $\mathrm{p}^{\text {th }}$ order exponential distribution and its characterization, J. Statist. Res. Iran 7, 201-212.

[10] Mohie El-Din, M. M., Mahmoud, M. A. W., Abu-Youssef, S. E. and Sultan, K. S. (1997). Order statistics from the doubly truncated linear exponential distribution and its characterizations. Commun. Statist.- Simul. Comput. 26, 281-290.

[11] Nain, K. (2010 a). Recurrence relations for single and product moments of $\mathrm{k}^{\text {th }}$ record values from generalized Weibull distribution and a characterization. International Mathematical Forum, 5, No. 33, 1645-1652.

[12] Nain, K. (2010 b). Recurrence relations for single and product moments of ordinary order statistics from $\mathrm{p}^{\text {th }}$ order exponential distribution. International Mathematical Forum, 5, No. 34, 1653 1662.

[13] Pawlas, P. and Szynal, D. (2001). Recurrence relations for single and product moments of generalized order statistics from Pareto, Generalized Pareto and Burr distributions. Commun. Statist. Theor. Meth., 30, 739-746.

[14] Saran, J. and Nain, K. (2012a). Recurrence relations for single and product moments of generalized order statistics from doubly truncated $\mathrm{p}^{\text {th }}$ order Exponential Distribution, JKSA 23.
[15] Saran, J. and Nain, K. (2012b). Relationships for moments of kth record values from doubly truncated pth order exponential and generalized Weibull distributions. ProbStat Forum., 05, 142-149.

[16] Saran, J. and Nain, K. (2012c). Relationships for moments of kth record values from doubly truncated pth order exponential and generalized Weibull distributions. ProbStat Forum., 05, 142-149.

[17] Saran, J and Nain, K (2013). Explicit Expressions for Single and Product Moments of Generalized Order Statistics from a New Class of Exponential Distributions Characterization, JKSA 24, 37 52.

[18] Saran, J. and Pandey, A. (2004). Recurrence relations for single and product moments of generalized order statistics from linear exponential distribution. Journal of Applied Statistical Science, 13, 323-333.

[19] Saran, J. and Pandey, A. (2009). Recurrence relations for single and product moments of generalized order statistics from linear exponential and Burr distributions. Journal of Statistical Theory and Applications, 8, No. 3, 383-391.

[20] Saran, J. and Pandey, A. (2011). Recurrence relations for marginal and joint moment generating functions of dual generalized order statistics from inverse Weibull distribution. Journal of Statistical Studies, 30, 65-72.

[21] Saran J. and Pande V.(2012). Recurrence relation for moments of progressively type-II right censored order statistics from half logistic distribution. J. Statistical Theory and Applications, 11, 8796.

[22] Saran, J. and Pushkarna, N. (1999). Moments of order statistics from doubly truncated linear exponential distribution. J. Korean Statist. Soc., 28, 279-296. 